\title{
Percepción de la satisfacción con la labor desempeñada y factores de motivación del personal de las instituciones prestadoras de servicios de salud (IPS) del valle de Aburrá, 2011
}

\author{
Perception of the Performed Work Satisfaction and Motivational Factors among the Personnel of the \\ Healthcare Service Suppliers in the Aburrá Valley during 2011 \\ Percepção de satisfação com o trabalho executado e fatores de motivação da equipe das instituiçóes \\ prestadoras de serviços de saúde (IPS) do vale do Aburrá, 2011
}

Gladys Irene Arboleda Posada ${ }^{\mathrm{a}}$

Corporación Universitaria Americana, Facultad Nacional de Salud Pública, Universidad de Antioquia, Colombia gladysarboledap@yahoo.com.co

ORCID: http://orcid.org/0000-0003-3690-2842

Jairo León Cardona Jiménez

Corporación Universitaria Remington, Universidad de Antioquia, Colombia

ORCID: http://orcid.org/0000-0003-3383-1401
DOI: https://doi.org/10.11144/Javeriana.rgps17-35.psld Redalyc: http://www.redalyc.org/articulo.oa?id=54557477011

Fecha de recepción: 30 Agosto 2017 Fecha de aprobación: 20 Abril 2018 Fecha de publicación: 01 Noviembre 2018

\section{Resumen:}

Introducción: el conocer las variables que influyen en el desempeño laboral permite ofrecer aquellos elementos que contribuyan a lograr la satisfacción laboral. Objetivo: examinar la percepción de la satisfacción con la labor desempeñada y los factores de motivación del personal de las instituciones prestadoras de servicios de salud (IPS) del valle de Aburrá, Colombia, en el año 2011. Materiales y métodos: estudio de corte transversal. La población fue el personal que laboraba en estas instituciones, el total de la muestra fue de 224 empleados y 69 instituciones elegidas de manera aleatoria. Resultados: $52,2 \%$ de los encuestados expresaron sentirse muy satisfechos con la labor que desempeñan en la institución y sentirse motivados, principalmente, por el ambiente de trabajo (70,5\%), seguido por el salario y el cargo, con igual participación (49,6\%); también mayor satisfacción con el trabajo realizado en los empleados asistenciales en comparación con los administrativos $(\mathrm{p}=0,018)$. Entre los factores menos motivacionales se encontraron: las posibilidades de ascenso, bonificaciones y reconocimientos. Conclusión: gran parte de los empleados se sienten satisfechos con la labor desempeñada, y se destaca como principal factor motivador el ambiente de trabajo. Palabras clave: satisfacción en el trabajo, condiciones de trabajo, ambiente de trabajo, salarios y beneficios.

\begin{abstract}
:
Introduction: Knowing the variables influencing the labor performance allows to provide the elements that will contribute to the work satisfaction. Objective: To examine the perception of the performed work satisfaction as well as the motivational factors among the personnel of the healthcare service suppliers in the Aburrá Valley, Colombia during 2011. Materials and Methods: This is a cross-sectional study and the population studied was the personnel working in the said companies. The total sample included 224 employees and 69 institutions randomly selected. Results: $52.2 \%$ of the interviewees stated to feel very satisfied with the work they performed at the institution and felt motivated mostly by the working environment (70.5\%) and then by the wage and the position in equal percentages (49.6\%). It was also found a greater satisfaction due to the work performed by the assistant employees as compared to those in managerial positions $(\mathrm{p}=0.018)$. The less motivational factors included: the opportunities for promotion, bonuses and acknowledgements. Conclusion: A great deal of employees feel satisfied with their performed work and the working environment stands out as the main motivational factor.
\end{abstract}

Keywords: satisfaction at work, working conditions, work environment, wages and benefits. 


\section{Resumo:}

Introdução: conhecer as variáveis que influenciam o desempenho laboral permite oferecer aqueles elementos que contribuem para a satisfação laboral. Objetivo: examinar a percepção da satisfação com o trabalho executado e os fatores de motivação do pessoal das instituições prestadoras de serviços de saúde (IPS) no vale do Aburrá, Colômbia, em 2011. Materiais e métodos: estudo de corte transversal. A população foi a equipe que laborava nestas instituições, o total da amostra foi de 224 funcionários e 69 instituições eleitas de forma aleatória. Resultados: $52,2 \%$ dos perguntados expressaram se sentir muito satisfeitos com o trabalho executado na instituição e se sentir motivados, principalmente, pelo ambiente de trabalho (70,5\%), seguido pelo salário e o cargo, com igual participação (49,6\%); mesmo maior satisfação com o trabalho realizado nos trabalhadores assistenciais em comparação com os administrativos $(p=0,018)$. Entre os fatores menos motivacionais encontraram-se: as possibilidades de promoção, bônus e reconhecimentos. Conclusão: grande parte dos funcionários sente-se satisfeita com o trabalho executado e o ambiente de trabalho se destaca como principal fator motivador.

Palavras-chave: satisfação no trabalho, condições de trabalho, ambiente de trabalho, salários e benefícios.

\section{Introducción}

El personal representa el capital humano de una organización, conocer las necesidades reales o sentidas que influyen en su desempeño laboral es una prioridad que permite ejecutar acciones tendientes a ofrecer a los trabajadores aquellos elementos que contribuyan a lograr la satisfacción en el desempeño de sus funciones, lo cual se verá reflejado en la productividad y el ambiente de trabajo (1). La satisfacción laboral en los servicios de salud está implicada en la calidad asistencial, de ahí la importancia de su medición en los trabajadores de las instituciones sanitarias (1).

El bienestar laboral se da por la combinación de factores como el ingreso para el cubrimiento de necesidades básicas individuales y del grupo familiar, el reconocimiento institucional y tener estabilidad laboral; además, poder capacitarse, ingresar a la educación superior y desarrollarse profesionalmente (2). Según un estudio realizado por el Banco Interamericano de Desarrollo (BID) (3), 81\% de los latinoamericanos están satisfechos con su empleo, satisfacción mayor que la de los trabajadores de países con ingresos superiores como Japón y Corea del Sur.

La insatisfacción del personal puede llevar a una disminución en la calidad de trabajo, ineficiencia en procesos y procedimientos internos de los empleados, pérdida del sentido de pertenencia o dispersión en las funciones asignadas, aspectos que repercutirían en la calidad de atención para el usuario externo (4). Es necesario que los empleados encuentren en su trabajo no solo la fuente para satisfacer sus necesidades económicas básicas, sino que sea el lugar adecuado para satisfacer otras necesidades, como autoestima y realización, tanto personal como profesional; que sea el espacio de trabajo donde puedan crecer y desarrollar sus capacidades y potencialidades. Es responsabilidad de los administradores de las instituciones de salud crear las condiciones adecuadas para que sus empleados se sientan parte integrante de la institución, "que se identifiquen con ella y se sientan y actúen como responsables por el logro de sus objetivos” (5).

En los resultados del trabajo de Arboleda (4), los encuestados, personal de salud de tercer nivel de atención, afirman en su gran mayoría (93\%) que están muy satisfechos o satisfechos con la labor que desempeñan en la institución. Al precisar en relación con la antigüedad en la institución, están más satisfechos los que cuentan entre 10 y 20 años de antigüedad, con el 95\%, contra el 86\% en quienes llevan más de 20 años de servicio. En general, se percibe que a mayor edad disminuye el grado de satisfacción, pero se destaca el bajo reconocimiento por su trabajo, pues el $46 \%$ opina que nunca o casi nunca ha obtenido reconocimientos; igualmente, el $45 \%$ considera que los ascensos nunca se hacen por méritos.

Pérez-Zapata et al. (6) reportan estudios realizados respecto a calidad de vida laboral en funcionarios del área de la salud y señalan la presencia de una baja percepción de esta, junto a una pobre motivación intrínseca, lo que repercute en las fortalezas y debilidades de los ambientes de trabajos. Y agregan: "Existe evidencia de que la presencia de variables de gestión como sobrecarga laboral, salario y escasez de tiempo disponible influyen desfavorablemente en la sensación de bienestar experimentada por los trabajadores" y repercuten en su rendimiento laboral y su estado de salud, al asociarse a la presencia de altos niveles de ansiedad $(1,7)$. 
Estos mismos autores encontraron diferencias significativas en la percepción de calidad de vida laboral en función del cargo de los participantes. Observaron diferencias en las valoraciones subjetivas entre los sujetos con cargos directivos y técnicos y señalaron que estas diferencias podrían ser explicadas por la estructura organizacional de los servicios de salud pública de Chile, los cuales en sus estamentos técnicos tienen una gran carga laboral, labores repetitivas con un contacto permanente con el usuario de salud; además, realizan sus funciones con insuficientes insumos asistenciales y pocas comodidades en infraestructura, lo que se relaciona con una baja percepción de calidad de vida laboral (6).

En el trabajo de Ma. Guadalupe Nava-Galán et al. (8), realizado en cuatro instituciones de México, los factores mejor calificados fueron la relación interpersonal con jefes y compañeros, mientras que los peor valorados fueron la competencia y la promoción profesional. Refiere la autora que los aspectos intrínsecos marcan la diferencia. Como el personal se encuentra satisfecho con la labor que realiza, evalúa como buenas las relaciones con sus superiores y compañeros, pero considera limitados los aspectos de promoción y competencia laboral.

Arias Jiménez (9) midió la satisfacción laboral de enfermería en una unidad de cuidados intensivos neonatales y resaltó la importancia del clima laboral y de la realización exitosa del trabajo, por encima de otros factores, en la satisfacción laboral. Entre los factores con bajo puntaje halló el de promoción profesional, la presión en el trabajo y la tensión relacionada con este. Parra y Paravic (10) encontraron que los profesionales de enfermería opinan que las oportunidades de promoción y ascenso son muy limitadas. Resultados similares fueron reportados en la revisión hecha por Río del Moro et al. (11), donde se concluye que la no promoción es uno de los aspectos del trabajo que más insatisfacción producen entre los profesionales de enfermería.

Salvador Juárez-Adauta (12) expresa que a mayor satisfacción laboral mejor clima organizacional. Además, no encontró diferencias significativas en cuanto a la categoría de trabajo, pero en los trabajadores de servicios básicos, seguido por enfermería y el personal de asistencia técnica y personal administrativo, su nivel fue menor. Tampoco encontró correlación significativa entre el nivel de satisfacción laboral y la antigüedad institucional, lo que significa que la antigüedad no muestra relación con el nivel de satisfacción laboral ni con el clima organizacional. No se encontraron diferencias según sexo. Respecto al cargo, reportó mayor frecuencia de médicos, enfermeras y personal de asistencia técnica con alto nivel de satisfacción laboral. En el personal administrativo, entre nivel medio y alto, y una gran proporción del personal de servicios básicos con menores niveles, a mayor antigüedad las opiniones sobre la satisfacción laboral y el clima organizacional se dividieron (12).

En el estudio de Carrillo et al. (13) sobre profesionales sanitarios adscritos a un hospital universitario público, los resultados reflejan una satisfacción general media, los "compañeros de trabajo" y el "superior inmediato" son los aspectos mejor evaluados. Se encuentran diferencias en los niveles de satisfacción de acuerdo con la categoría profesional, siendo los más satisfechos los grupos de gestión y los médicos residentes. En cambio, los facultativos especialistas de área, profesionales y auxiliares de enfermería manifiestan niveles de satisfacción más reducidos.

Según Cifuentes (14), los hombres enfermeros se hallan más satisfechos con su trabajo, pero acusan mayor presión y tensión. En ambos sexos, quienes han laborado en su profesión por más de siete años se encuentran más insatisfechos que aquellos que llevan un tiempo menor. Se encontraron como factores protectores el servicio en el cual los profesionales de enfermería cumplen sus actividades, junto con la asistencia a cursos de educación continuada, frente a la insatisfacción con el trabajo. No se hallaron relaciones estadísticamente significativas entre la satisfacción laboral de los profesionales de enfermería en relación con el sexo, la edad, la formación académica y el servicio al cual se hallan asignados.

En el estudio de Arboleda (15), las necesidades del trabajador y de su grupo familiar son cubiertas satisfactoriamente por el ingreso recibido en el 61\%, mientras que en México Cabrera et al. (16) reportan el $29 \%$. 
En el trabajo de Herrera y Manrique (17) con profesionales de enfermería, la remuneración se encontró diferente según el tipo de contratación: hay mayor satisfacción en el grupo de profesionales contratados indefinidamente y la estabilidad dada por el tipo de contrato favorece la satisfacción.

En otro trabajo, realizado con personal médico en el servicio de urgencias adultos en un hospital general regional del Perú (18), se encontró un nivel medio de satisfacción laboral, donde la poca disponibilidad de recursos fue el principal factor.

Los incentivos son medios importantes que las organizaciones pueden utilizar para atraer, retener, motivar, dar satisfacción y mejorar los resultados del personal. Se emplean de manera generalizada en las organizaciones, tanto en el sector público como en el privado, en todos los contextos laborales, según informe del Consejo Internacional de Enfermeras y Otros (19), y llevan a los encargados de la política general y a los gestores a utilizar incentivos para mejorar la contratación, la motivación laboral. Por esta se entiende el estado interno relacionado con aquellos factores que pueden dirigir la conducta hacia un objetivo y afectar el nivel o la intensidad del comportamiento, a diferencia de la satisfacción laboral que se refiere al estado emocional positivo que proviene de la percepción subjetiva de las experiencias laborales, y determina el balance final entre el rol que cada trabajador quiere cumplir y el que finalmente desempeña (20). Y, por tanto, relacionada con las actitudes hacia el trabajo en general o a facetas de este (21).

De acuerdo con lo anterior, el presente trabajo busca examinar la percepción de la satisfacción con la labor desempeñada y los factores de motivación del personal de las instituciones prestadoras de servicios de salud (IPS) del valle de Aburrá Colombia, en el 2011.

\section{Materiales y métodos}

Estudio de corte transversal. La ejecución se llevó a cabo mediante la aplicación de encuestas en las instituciones públicas y privadas de alta y mediana complejidad en el valle de Aburra, Colombia, en el 2011. De un total de 374 instituciones, fueron elegidas aleatoriamente 69, con una muestra de 224 empleados, lo que aseguraba que estuvieran incluidos ambos niveles de atención y los sectores público y privado.

En el análisis de las variables sociodemográficas y formación académica y laboral del encuestado se calcularon porcentajes y pruebas Chi cuadrado en tablas de contingencia, para determinar la relación entre las variables. En todos los casos, se consideró significativo cualquier valor p menor de 0,05.

El instrumento fue aplicado por el investigador principal y dos estudiantes de último semestre del programa de Administración en Salud, previamente capacitados, con compresión de la terminología empleada y habilitados para explicar las dudas de los encuestados. El instrumento se aplicó de manera individual y personal.

El contenido de las preguntas fue desarrollado a partir de varios trabajos realizados acerca de temáticas relacionadas $(15,22,23)$. Con el fin de verificar la eficacia de la encuesta, se realizó la prueba piloto en una IPS que corroboró su validez. El tipo de preguntas fue estructurado: ¿cómo se siente con la labor desempeñada en la institución? (muy satisfecho, satisfecho, ni satisfecho ni insatisfecho, insatisfecho, muy insatisfecho); ¿qué lo motiva en la institución para el desempeño de sus labores (puede elegir varias opciones): salario, reconocimiento, ambiente de trabajo, cargo, bonificaciones, posibilidad de acenso y promoción institucional, ¿otros?, ¿cuáles?

Los criterios de inclusión fueron: personal que laboraba en IPS de mediana y alta complejidad ubicadas en el valle de Aburrá al momento de aplicación de la encuesta, con más de un año de laborar en la institución, sin importar el tipo de contrato, con formación técnica, tecnológica, profesional, especialista, magíster o doctorado. Se excluyeron los empleados con cargos directivos de primero y segundo rango, de acuerdo con cada estructura orgánica. 
Consideraciones éticas: la información recibida fue de carácter confidencial, se utilizó únicamente para fines académicos y dar cumplimiento a los objetivos propuestos. Se garantizó la omisión de los nombres de los encuestados. Las preguntas hechas no tocaron la intimidad, se contó con el consentimiento informado, según lo establece la Resolución 8430 de 1993 del Ministerio de Salud de Colombia (24), y la aprobación por el Comité de Bioética de la Facultad Nacional de Salud Pública de la Universidad de Antioquia en el Acta 049 de marzo del 2011, donde se da el aval en categoría riesgo mínimo.

\section{Resultados}

El personal entrevistado fue en gran proporción del sexo femenino (79,9\%), el rango de edad más frecuente fue entre 30 y 49 años (57\%). Respecto al nivel educativo, se encontraron técnicos o tecnólogos (54\%), educación profesional (31,3\%). Un 12\% realizó algún posgrado y la cuarta parte de estos son asistenciales. De acuerdo con el tipo de vinculación, el 52\% tiene contrato a término indefinido, el 31\% a término fijo, y solo el 3\% labora con la modalidad de cooperativa de trabajo asociado.

Con relación a la satisfacción con la labor desempeñada, el sexo femenino expresó mayor satisfacción $(54,2 \%)$ que el masculino (44,4\%) (diferencias no significativas). En referencia al tipo de cargo, los asistenciales manifestaron estar muy satisfechos con la labor desempeñada en la institución (58,7\%), en comparación con el 41,3\% en los cargos administrativos (diferencias significativas $\mathrm{p}=0,018$ ). Cabe destacar que no se presentaron manifestaciones de insatisfacción (figura 1).

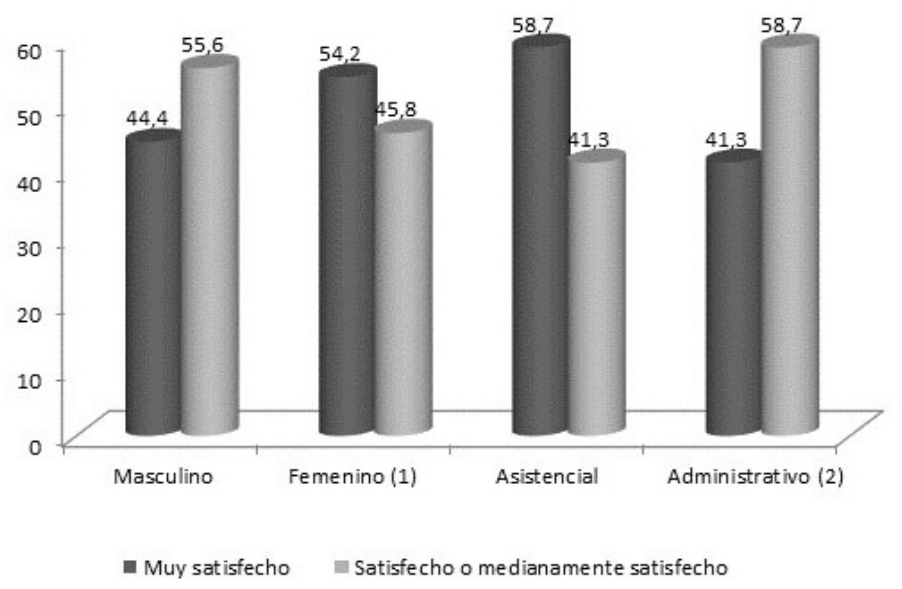

(1) $X^{2}=1,006, g l=1, p=0,316,0$ casillas $(0,0 \%)$ tienen una frecuencia esperada inferior a 5 , son independientes (2) $X^{2}=6,612, g l=1, p=0,018,0$ casillas $(0,0 \%)$ tienen una frecuencia esperada inferior a 5 , son dependientes

FIGURA 1

Satisfacción con la labor desempeñada según sexo tipo de cargo. Fuente: elaboración propia.

Con el grado de muy satisfechos se destacaron la totalidad de los subdirectores asistenciales, seguidos de las enfermeras y profesionales asistenciales de otras áreas; con los menores porcentajes estaban los odontólogos y los subdirectores administrativos (tabla 1 ). 
TABLA 1

Satisfacción con la labor desempeñada según cargo

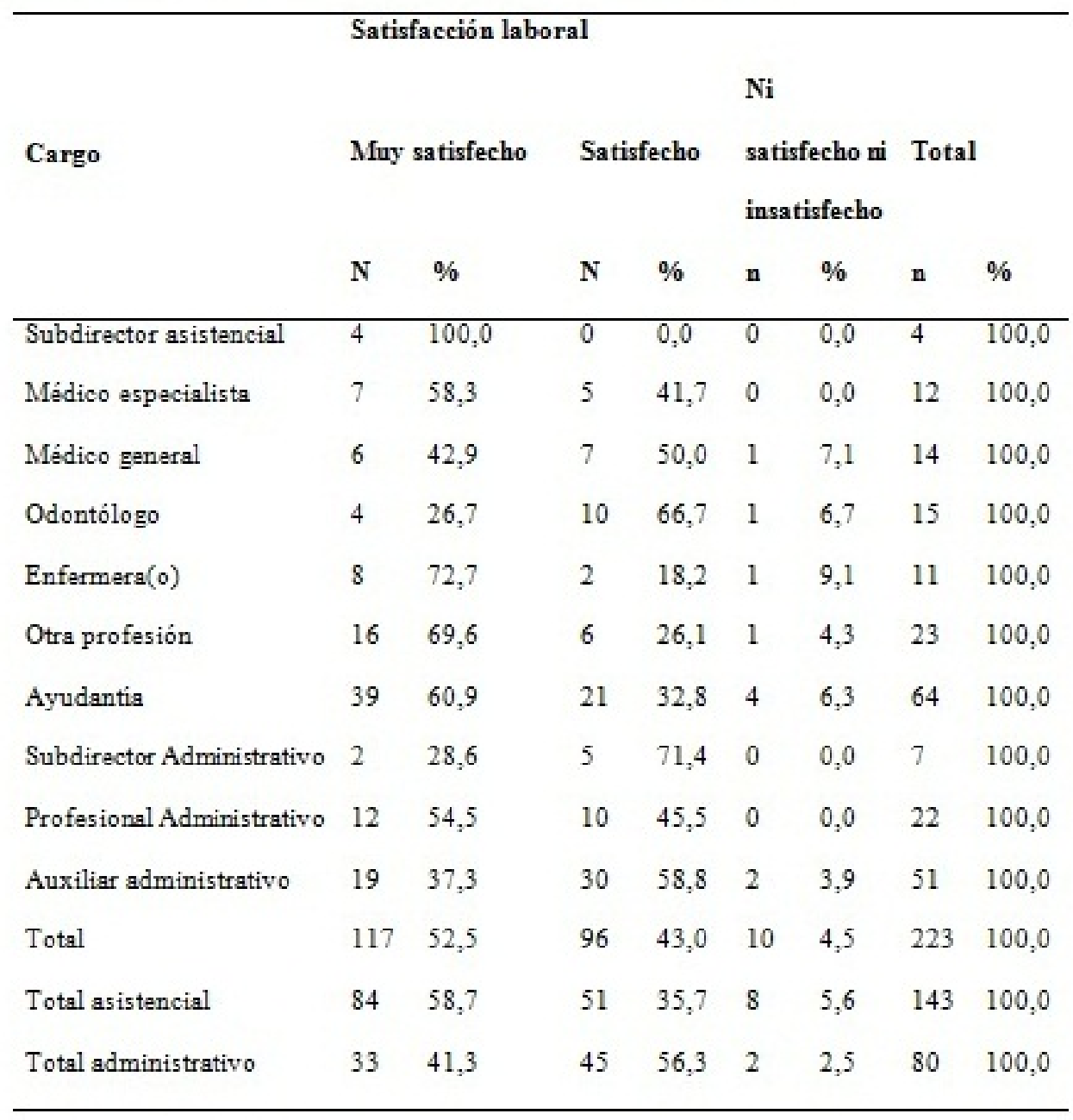

Fuente: elaboración propia.

En cuanto a la relación entre edad, antigüedad en la institución y nivel de ingresos, no se encontró asociación significativa en la satisfacción con la labor desempeñada (tabla 2). En referencia a la pregunta sobre factores de motivación para el desempeño de sus labores, el personal manifestó que los factores que más lo motivaban eran: el ambiente de trabajo $(70,5 \%$, ) seguido del salario y el cargo $(49,6 \%$, ambos factores con igual proporción). Aspectos como bonificaciones, posibilidades de ascenso y promociones tuvieron los menores porcentajes (figura 2). 
TABLA 2

Satisfacción con la labor desempeñada según la edad, la antigüedad y el ingreso en salarios mínimos legales

\begin{tabular}{|c|c|c|c|c|c|c|c|c|}
\hline \multirow{3}{*}{ Satisfacción laboral } & \multicolumn{8}{|c|}{ Edad en aíos (1) } \\
\hline & \multicolumn{2}{|c|}{$18-29$} & \multicolumn{2}{|c|}{$30-49$} & \multicolumn{2}{|c|}{500 más } & \multicolumn{2}{|c|}{ Total } \\
\hline & n & $\%$ & n & 90 & $\mathrm{n}$ & $\%$ & n & $\%$ \\
\hline Muy satisfecho & 36 & 49,3 & 66 & 52,4 & 13 & 59,1 & 115 & 52,0 \\
\hline $\begin{array}{l}\text { Satisfecho o medianamente } \\
\text { satisfecho }\end{array}$ & 37 & 50,7 & 60 & 47,6 & 9 & 40,9 & 106 & 48,0 \\
\hline Total & 73 & 100,0 & 126 & 100,0 & 22 & 100,0 & 221 & 100,0 \\
\hline & \multicolumn{8}{|c|}{ Antigüedad en aivos (2) } \\
\hline & \multicolumn{2}{|c|}{$1-5$} & \multicolumn{2}{|c|}{$6-10$} & \multicolumn{2}{|c|}{ Más de 10} & \multicolumn{2}{|c|}{ Total } \\
\hline Muy satisfecho & 74 & 48,4 & 19 & 61,3 & 24 & 60,0 & 117 & 52,2 \\
\hline $\begin{array}{l}\text { Satisfecho o madianamente } \\
\text { satisfecho }\end{array}$ & 79 & 51,6 & 12 & 38,7 & 16 & 40,0 & 107 & 47,8 \\
\hline \multirow[t]{3}{*}{ Total } & 153 & 100,0 & 31 & 100,0 & 40 & 100,0 & 224 & 100,0 \\
\hline & \multicolumn{8}{|c|}{ Ingreso (en salarios minimos) (3) } \\
\hline & \multicolumn{2}{|c|}{$1-3$} & \multicolumn{2}{|c|}{ Más de 3 - 6} & \multicolumn{2}{|c|}{60 más } & \multicolumn{2}{|c|}{ Total } \\
\hline Muy satisfecho & 66 & 52,4 & 34 & 54,0 & 11 & 55.0 & 111 & 53,1 \\
\hline $\begin{array}{l}\text { Satisfecho o madianamente } \\
\text { satisfecho }\end{array}$ & 60 & 47,6 & 29 & 46,0 & 9 & 45,0 & 98 & 46,9 \\
\hline Total & 126 & 100,0 & 63 & 100,0 & 20 & 100,0 & 209 & 100,0 \\
\hline
\end{tabular}

(1) $\mathrm{X}^{2}=0,661, \mathrm{gl}=2, \mathrm{p}=0,718$, 0 casillas $(0,0 \%)$ tienen una frecuencia esperada inferior a 5 ; son independientes.

(2) $X^{2}=1,499, g l=2, p=0,473$, 0 casillas $(0,0 \%)$ tienen una frecuencia esperada inferior a 5 ; son independientes.

(3) $\mathrm{X}^{2}=0,074, \mathrm{gl}=2, \mathrm{p}=0,964$, 0 casillas

$(0,0 \%)$ tienen una frecuencia esperada inferior a 5 ; son independientes.

Fuente: Elaboración propia.

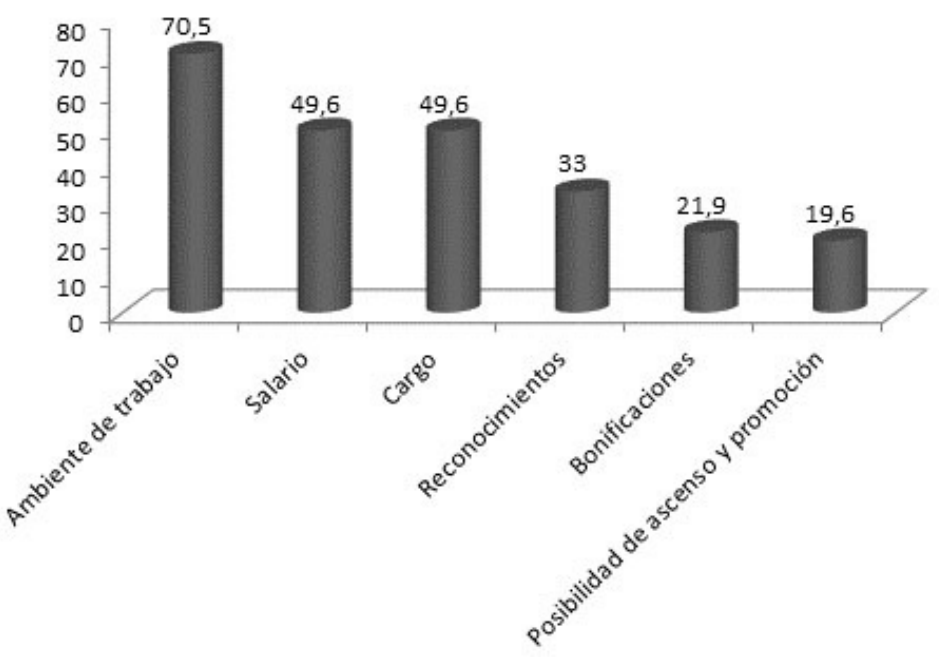

FIGURA 2

Distribución porcentual de la motivación en la institución para el desempeño de sus labores. Fuente: elaboración propia.

$\mathrm{Al}$ analizar los factores de motivación por sexo, se observó que en ambos el ambiente de trabajo tuvo el mayor porcentaje, seguido en los hombres por el salario y el cargo, en las mujeres por el cargo y el salario. Los valores más bajos en ambos sexos fueron la posibilidad de ascenso y promoción con porcentajes muy similares (tabla 3). 
TABLA 3

Motivación para el desempeño de labores según sexo

\begin{tabular}{llcllll}
\hline Motivación para el desempeño de & \multicolumn{2}{l}{ Sexo } & & & \\
labores(a) & \multicolumn{2}{l}{ Masculino } & Femenino & Total \\
& N & $\%$ & $\mathbf{n}$ & $\%$ & $\mathbf{n}$ \\
\hline Salario & 24 & 54,5 & 87 & 51,8 & 111 \\
Reconocimientos & 20 & 45,5 & 54 & 32,1 & 74 \\
Ambiente de trabajo & 34 & 77,3 & 124 & 73,8 & 158 \\
Cargo & 23 & 52,3 & 88 & 52,4 & 111 \\
Bonificaciones & 11 & 25,0 & 38 & 22,6 & 49 \\
Posibilidad de ascensoy promoción & 9 & 20,5 & 35 & 20,8 & 44 \\
Total & 45 & & 168 & & 212 \\
\hline
\end{tabular}

Los porcentajes y los totales se basan en los encuestados. Tabulado el valor 1.

Fuente: elaboración propia.

El personal asistencial expresó mayor motivación por factores como ambiente de trabajo, cargo y salario, en su orden. En los cargos administrativos también se encontró el ambiente de trabajo como principal factor, seguido del salario. En ambos grupos, los de menor puntaje correspondieron a las bonificaciones y la posibilidad de ascenso y promoción (figura 3).

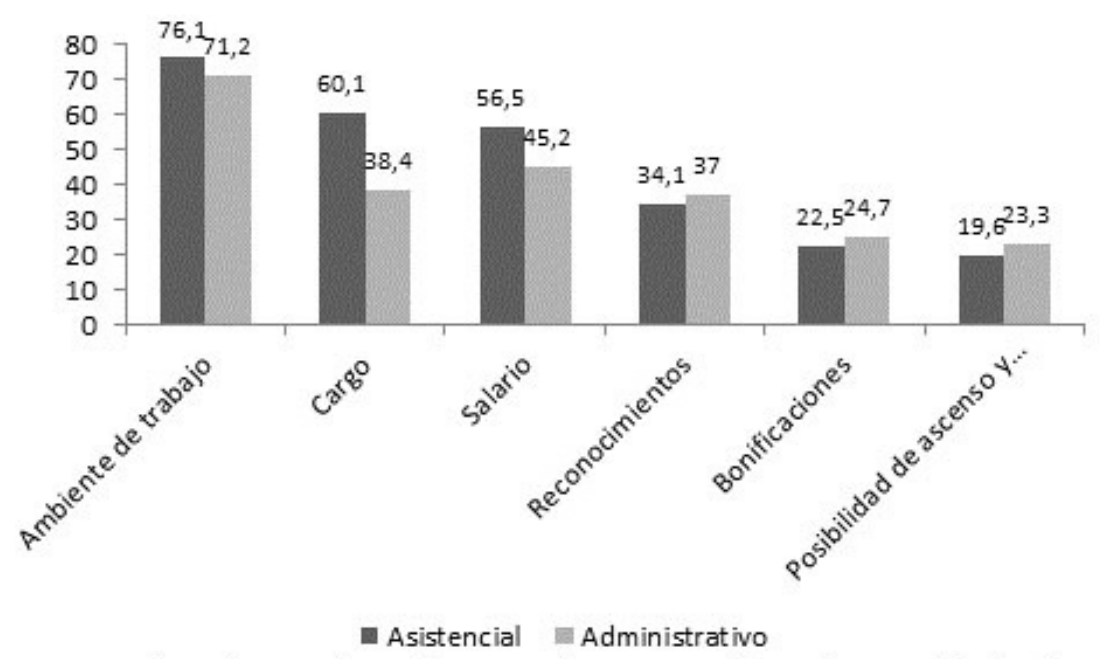

FIGURA 3

Motivación para el desempeño de labores según Tipo de cargo

Fuente: elaboración propia.

Según el nivel de ingreso, los factores motivacionales mayores para quienes ganaban más de seis salarios mínimos fueron: el salario, el ambiente de trabajo y el cargo, en su orden. Para quienes ganan de tres a seis salarios, son el ambiente de trabajo, los salarios y el cargo en su orden; para los de menores ingresos, el factor más motivador fue el ambiente de trabajo, seguido del cargo y el salario respectivamente (tabla 4) 
TABLA 4

Motivación para el desempeño de labores según ingreso

\begin{tabular}{|c|c|c|c|c|c|c|c|}
\hline \multirow{3}{*}{ Motivación por } & \multicolumn{7}{|c|}{ Ingreso (en salarios mínimos) } \\
\hline & \multicolumn{2}{|c|}{$1-3$} & \multicolumn{2}{|c|}{ Más de 3 - 6} & \multicolumn{2}{|c|}{60 más } & \multirow{2}{*}{$\begin{array}{l}\text { Total } \\
\text { n }\end{array}$} \\
\hline & $\mathbf{N}$ & $\%$ & $\mathbf{N}$ & $\%$ & $\mathbf{n}$ & $\%$ & \\
\hline Salario & 50 & 42,4 & 39 & 63,9 & 15 & 78,9 & 104 \\
\hline Reconocimientos & 34 & 28,8 & 26 & 42,6 & 8 & 42,1 & 68 \\
\hline Ambiente de trabajo & 90 & 76,3 & 44 & 72,1 & 13 & 68,4 & 147 \\
\hline Cargo & 54 & 45,8 & 38 & 62,3 & 12 & 63,2 & 104 \\
\hline Bonificaciones & 24 & 20,3 & 16 & 26,2 & 5 & 26,3 & 45 \\
\hline $\begin{array}{l}\text { Posibilidad de } \\
\text { ascenso y promoción }\end{array}$ & 23 & 19,5 & 13 & 21,3 & 3 & 15,8 & 39 \\
\hline Total & 118 & & 61 & & 19 & & 189 \\
\hline
\end{tabular}

Los porcentajes y los totales se basan en los encuestados. Tabulado el valor 1 .

Fuente: elaboración propia.

\section{Discusión}

A continuación, se discutirán los resultados más relevantes en comparación con otros estudios relacionados.

En general, los encuestados se sienten muy satisfechos con la labor que desempeñan en la institución. En referencia al tipo de cargo, en este estudio se encontraron diferencias significativas entre quienes ocupan cargos asistenciales, donde está muy satisfecho el 58,7\%, en contraste con el 41,3\% en los administrativos; Juarez Aduta (12) tambien halló menor satisfacción en administartivos y servicios generales. A su vez, Carrillo et al. (13) encontraron diferencias atendiendo a la categoría profesional, siendo los grupos de gestión y los médicos residentes lo más satisfechos, mientras que los especialistas de área, profesionales de enfermería y auxiliares de enfermería manifiestan niveles más bajos. Pérez et al. (6) encontraron en Chile diferencias entre los estamentos directivos y los técnicos que atribuyeron a las desigualdades en la estructura organizacional en los servicios de salud publica del pais.

En cuanto al sexo, no se hallaron diferencias significativas en la satisfacción con la labor desempeñada. Resultados similares fueron reportados por Cifuentes (14) y Juárez (12). A propósito de la edad, los hallazgos no supusieron diferencias significativas, aunque en este estudio es mayor a medida que esta aumenta. Herrera y Manrique (17) obtuvieron resultados diferentes; hallaron mayor satisfacción en el grupo de edad de 32 a 40 años. Cifuentes (14) tampoco encontró diferencias en relación con la edad, pero sí en quienes llevaban más de siete años de servicio, quienes reportaron menor satisfacción. Los resultados son contrarios a los hallados en este estudio, donde quienes llevan más de seis años en la institución tienen mayor satisfacción que los de menor tiempo de servicio (diferencias no significativas). 
El 70,5\% de los empleados expresó sentirse motivado principalmente por el ambiente de trabajo. Este parece ser el principal factor, pues es reportado en varios estudios en el contexto latinoamericano $(6,8$, 9,13), pero se refieren otros trabajos donde el nivel de satisfacción no es alto, como el de Chavarría-Islas et al. (18), realizado con personal médico del servicio de urgencias. En este caso, si bien la mayoría del personal considera que existe un ambiente de trabajo adecuado, para el desempeño de sus labores en los indicadores de respeto, confianza y reconocimiento, el principal factor condicionante de insatisfacción por parte del personal es la poca disponibilidad de recursos para el desempeño laboral. En otro estudio, realizado con personal de enfermería con más de 20 años de servicio, el grado de satisfacción fue del 58\%, y la promoción profesional y el reconocimiento por su trabajo fue el factor que presentó un mayor valor de correlación con la satisfacción laboral (25). En el presente estudio los resultados fueron; estos factores mostraron menores porcentajes.

En segundo lugar está el salario. En el mismo sentido, Borra y Gómez (26) reportan el salario como exógeno en la ecuación de satisfacción laboral y tiene un efecto significativamente positivo sobre esta. Sin embargo, en el estudio de Chiang (27) este factor aparece en quinto lugar, antecedido por la satisfacción en la relación con sus superiores, con las condiciones físicas, la participación en las decisiones y la satisfacción con su trabajo. Pérez et al. (6) lo consideran uno de los factores, junto con la sobrecarga laboral y el poco tiempo para la realización de sus labores, que influyen desfavorablemente en la sensación de bienestar.

De igual manera, los enfermeros se encuentran menos satisfechos con las promociones y remuneraciones (28). Herrera y Manrrique (17) opinan que la autonomía de las EPS como intermediarias para contratar los servicios entre las IPS es una dificultad, especialmente para los profesionales no asociados u organizados previamente, que son la mayoría, y generalmente las nuevas promociones. Si en Colombia el objetivo de la reforma sigue siendo la reducción de los costos, corren mayor riesgo los salarios y las políticas de ascenso que deben ser percibidos como justos por parte de los empleados para sentirse satisfechos con su labor.

Es de resaltar que lo que menos los motiva es la posibilidad de ascensos y promociones; estos no necesariamente representan un aumento en el salario real, pero sí, en algunos casos, mayores responsabilidades y carga laboral, situación reportada por varios autores $(15,8,9,10)$.

Conscientes de que el factor más motivante es el ambiente de trabajo, y que este incide positivamente en la cultura organizacional, es importante que las instituciones lo fomenten, porque un apropiado ambiente laboral propiciará el aumento en iniciativas de proyectos, solución de problemas, mejoramiento de la comunicación interna, aumento de la competitividad en vía hacia una dirección más dinámica, garantizando el cumplimiento de las metas institucionales y elevando la productividad laboral. Jaramillo $e t$ al. (29) también hacen alusión a que el clima organizacional es un componente que permite la trasmisión de la cultura y el conocimiento, los cuales son factores claves del éxito.

Un buen ambiente de trabajo hace que el personal se sienta satisfecho, entendiendo la satisfacción con la labor desempeñada como un elemento que influye en la calidad de los servicios que oferta una institución, en la medida que su abordaje permite la identificación de problemas u oportunidades en el interior de la organización, lo cual mejora la ventaja competitiva como factor vinculante a la gestión del conocimiento (30).

La satisfacción laboral se ve impactada por factores como la infraestructura, la comunicación, el ambiente de trabajo, el salario, el cargo que se desempeña, entre otros. No es frecuente encontrar una persona satisfecha totalmente en el logro positivo de todos los factores, pero es tarea de la dirección procurar que la satisfacción laboral sea lo más alta posible, estableciendo condiciones internas para cubrir de manera integral las dimensiones de satisfacción laboral (14).

Lo anterior lo corroboran en su estudio Chiang et al. (27), al encontrar relación entre clima organizacional y satisfacción, en dimensiones como: "comunicación interna, reconocimiento, relaciones interpersonales, calidad en el trabajo, toma de decisiones, objetivos de la institución, compromiso, adaptación al cambio, delegación de actividades y funciones, coordinación externa y eficiencia de la productividad".

Molina et al. (31) destacan como factor motivante las buenas relaciones entre compañeros, las cuales aumentan el grado de satisfacción con el trabajo y protegen del desgaste profesional. Un trabajador 
insatisfecho genera un ambiente negativo entre sus compañeros y su entorno; además, es más propicio a las ausencias laborales, a los despidos, a renuncias voluntarias, a una posición agresiva e inconforme, a la insatisfacción y la disminución de la productividad en el proceso de atención.

Las instituciones tienen la responsabilidad social de crear condiciones favorables para el desarrollo de potencialidades de sus trabajadores, en un contexto de equidad, de oportunidades para la construcción de un proyecto de vida y, a través de este, el desarrollo de la institución.

La mayoría de los trabajos consultados deja ver poca satisfacción con los ascensos y las promociones, lo cual podría mejorarse mediante la participación de los trabajadores en tareas adicionales a la consulta, según lo proponen Carrillo et al. (13), para que obtengan un reconocimiento mediante la facilitación de la formación continuada u otras actividades, sin que supongan una sobrecarga a la actividad habitual del trabajador. Por otra parte, los puestos de responsabilidad, tanto directivos como organizativos, deberían definirse y reconocerse mediante incentivos monetarios, y no solo con un incremento de responsabilidades y carga laboral (15), además de poder disminuir la labor asistencial e invertir ese tiempo en realizar la tarea organizativa, aspecto que puede ser preferido por los profesionales al económico (13).

En resumen, los encuestados se sienten muy satisfechos con la labor que desempeñan en la institución y expresan sentirse motivados principalmente por el ambiente de trabajo, seguido por el cargo y el salario (estos últimos con igual porcentaje). También se encontró mayor satisfacción en los empleados asistenciales en comparación con los administrativos. Los factores que generan menor satisfacción son las posibilidades de ascenso, bonificaciones y reconocimientos.

\section{Conflicto de intereses}

Los autores declaran no tener ningún conflicto de intereses.

\section{Referencias bibliográficas}

1. García M, Luján ME, Martínez MÁ. Satisfacción laboral del personal de salud. Rev Enferm IMSS. 2007;15:63-72. Disponible en: https://www.new.medigraphic.com/cgi-bin/resumen.cgi?IDARTICULO=18895

2. Robles M, Dierssen T, Martínez E, Herrera P, Díaz A, Llorca J. Variables relacionadas con la satisfacción laboral: un estudio transversal a partir del modelo EFQM. Gac Sanit. 2005;19:127-34. DOI: https://www.dx.doi.org/ $10.1157 / 13074368$

3. Banco Interamericano de Desarrollo (BID). Mayor crecimiento económico afecta negativamente en las percepciones de la satisfacción de vida en América Latina y el Caribe [comunicado de prensa]. 2008. Disponible en: https://www.iadb.org/es/noticias/comunicados-de-prensa/2008-11-18/mayor-crecimiento-economico-af ecta-negativamente-en-las-percepciones-de-la-satisfaccion-de-vida-en-america-latina-y-el-caribe.4859.html

4. Arboleda G. Desarrollo humano en el personal vinculado a las instituciones de tercer nivel de atención: servicios de salud y bienestar laboral Medellín, Colombia 2007. Visión de Futuro 2011;15. Disponible en: https://www.re dalyc.org/articulo.oa?id=357935477005

5. Yépez F. El desarrollo humano y el éxito de las organizaciones. Cali: Instituto FES de Liderazgo, Programa de Gerencia Social; 1991.

6. Pérez D, Peralta J, Fernández P. Influencia de variables organizacionales en la calidad de vida laboral de funcionarios del sector público de salud en el extremo norte de Chile. Universitas Psychologica. 2014; 13(2):541-51. doi:10.11144/Javeriana. UPSY13-2.ivoc

7. Riveros J, Berné C, Múgica J. Gestión y satisfacción en servicios de salud de Chile: Contraste entre las percepciones de los funcionarios y los usuarios. Rev Med Chile. 2010;138:630-8. Disponible en: https://www.scielo.cl/pdf/ $\mathrm{rmc} / \mathrm{v} 138 \mathrm{n} 5 /$ art $15 . \mathrm{pdf}$

8. Nava MG, Hernández M, Hernández A, Pérez MT, Hernández MG, Matus R, et al. Satisfacción laboral del profesional de enfermería en cuatro instituciones de salud. Arch Neurocien. 2013;18(1):16-21. Disponible en: h ttps://www.medigraphic.com/pdfs/arcneu/ane-2013/anes131c.pdf 
9. Arias M. Factores del clima organizacional influyentes en la satisfacción laboral de enfermería, concerniente a los cuidados intensivos neonatales del Hospital Nacional de Niños, 2004. Enfermería en Costa Rica. 2007;28:12-9. Disponible en: https://bit.ly/jMzMu4

10. Parra $S$, Paravic T. Satisfacción laboral en enfermeras/os que trabajan en el sistema de atención médica de urgencia (SAMU). Cienc Enferm. 2002;8:37-48. Disponible en: https://www.dx.doi.org/10.4067/S0717-9553200200 0200005

11. Río O, Perezagua MC, Villalta MT, Sánchez A. Satisfacción personal de enfermería. ¿Se cumplen nuestras expectativas? Enfermería Cardiovascular [Internet] [citado 2015 abr. 23]. Disponible en: https://www.fac.org. ar/ccvc/llave/tl014/tl014.php

12. Juárez S. Clima organizacional y satisfacción laboral. Rev Med Inst Mex Seguro Soc. 2012;50:307-14. Disponible en: https://www.new.medigraphic.com/cgi-bin/resumen.cgi?IDARTICULO=38964

13. Carrillo C, Martínez M, Gómez C, Meseguer M. Satisfacción laboral de los profesionales sanitarios de un Hospital Universitario: análisis general y categorías laborales. Anales de Psicología. 2015;31:645-50. Disponible en: http s://www.dx.doi.org/10.6018/analesps.31.2.169791

14. Cifuentes JE. Satisfacción laboral en enfermería en una institución de salud de cuarto nivel de atención [tesis para optar el título de magíster en Enfermería]. Bogotá: Universidad Nacional de Colombia, Facultad de Enfermería, Programa de Maestría en Enfermería; 2012. Disponible en: https://www.bdigital.unal.edu.co/8907/1/539680 .2012.pdf

15. Arboleda GI. Ingreso y capacitación del personal vinculado a instituciones del tercer nivel de atención en salud. Medellín, Colombia. Rev Gerenc Polit Salud. 2012:11:134-51. Disponible en: https://www.scielo.org.co/pdf/ rgps/v11n23/v11n23a09.pdf

16. Cabrera CE, Franco SA, González G, Vega G, Parra J, Íñiguez JE. Satisfacción laboral de profesionales de la salud. Rev Med IMSS. 2004; 42:193-8. Disponible en: https://www.s2.medicina.uady.mx/observatorio/docs/pr/ac/ Pr2004_Ac_Cabrera.pdf

17. Herrera G, Manrique F. Condiciones laborales y grado de satisfacción de profesionales de enfermería. AQUICHAN. 2008;8:243-56. Disponible en: https://www.aquichan.unisabana.edu.co/index.php/aquichan/ article/view/150/286

18. Chavarría R, Sandoval E, Peláez K, Radilla CC. Satisfacción laboral del personal médico en el servicio de urgencias adultos en un hospital general regional. Revista CONAMED.2009;14:27-35.

19. Consejo Internacional de Enfermeras, Federación Farmacéutica Internacional, Federación Odontológica Mundial, Asociación Médica Mundial, Federación Internacional de Hospitales, Confederación Mundial de Fisioterapia. Directrices: incentivos para los profesionales de atención de salud. Ginebra, Suiza; 2008: Disponible en: https:/ /www.who.int/workforcealliance/documents/Incentives_Guidelines\%20SP.pdf

20. Marín H, Placencia M. Motivación y satisfacción laboral del personal de una organización de salud del sector privado. Horiz Med 2017; 17(4): 42-52. Disponible en: https://www.doi.org/10.24265/horizmed.2017.v17n 4.08

21. Chiang Vega MM, Salazar Botello CM, Martín Rodrigo MJ, Núñez Partido A. Clima organizacional y satisfacción laboral. Una comparación entre hospitales públicos de alta y baja complejidad. Salud de los Trabajadores (Maracay). [Internet]. 2011;19(1):5-16. Disponible en: https://www.redalyc.org/articulo.oa?id=37583929700 2

22. Molina G, Oquendo T, Rodríguez S, Montoya NC, Lagos Campos N, Almanza R, et al. Gestión del talento humano en salud pública. Un análisis en cinco ciudades colombianas, 2014. Rev Gerenc Polít Salud. 2016;15(30):108-25. Disponible en: https://www.dx.doi.org/10.11144/Javeriana.rgyps15-30.gtsp

23. Arboleda GI, Cardona JL. La investigación en instituciones de salud de mediana y alta complejidad del Valle de Aburrá, 2011. Rev Gerenc Polít Salud. 2015; 14(28):97-108. Disponible en: https://www.dx.doi.org/10.1114 4/Javeriana.rgyps18-28.liis

24. Colombia, Ministerio de Salud. Resolución número 8430 de octubre 4 de 1993. Por la cual se establecen las normas científicas, técnicas y administrativas para la investigación en salud. Bogotá: Ministerio de Salud; 1993. 
Disponible en: https://www.minsalud.gov.co/Normatividad_Nuevo/RESOLUCION\%208430\%20DE\%201 993.pdf

25. Contreras ML. Satisfacción laboral de los profesionales de enfermería vinculados a una I.P.S de III nivel de atención [tesis de maestría]. Bogotá: Universidad Nacional de Colombia; 2013. Disponible en: https://www.bdigital.un al.edu.co/43104/

26. Borra C, Gómez F. Satisfacción laboral y salario: ¿compensa la renta laboral las condiciones no monetarias del trabajo? Revista de Economía Aplicada. 2012;60:25-51. Disponible en: https://www.dialnet.unirioja.es/servle $\mathrm{t} /$ articulo? codigo $=4095686$

27. Chiang MM, Salazar CM, Núñez A. Clima organizacional y satisfacción laboral en un establecimiento de salud estatal: Hospital tipo 1. Theoría. 2007;16:1661-76. Disponible en: https://www.redalyc.org/articulo.oa?id=29 916206

28. Aguirre CD. Satisfacción laboral de los recursos humanos de enfermería. Factores que la afectan. Rev Haban Cienc Méd. 2009;8(4). Disponible en: http://www.scielo.sld.cu/scielo.php?script=sci_arttext\&pid=S1729-519X200 $9000400021 \& \operatorname{lng}=\mathrm{es}$

29. Jaramillo H, Latorre C, Lopera C, Albán C. El hospital como organización de conocimiento y espacio de investigación y formación. Bogotá: Editorial Universidad del Rosario; 2008.

30. Arboleda GI. Percepción de los gerentes acerca de la gestión del conocimiento en las instituciones de salud de mediana y alta complejidad del valle de Aburrá, 2011. Rev Fac Nac Salud Pública. 2014;32:42-53. Disponible en: https://www.scielo.org.co/scielo.php?script=sci_arttext\&pid=S0120-386X2014000200006\&lng=en

31. Molina JM, Avalos Martínez F, Valderrama LJ, Uribe AF. Factores relacionados con la satisfacción laboral de la enfermería de un hospital médico-quirúrgico. Invest Educ Enferm. 2009;27:218-25. Disponible en: https://ww w.scielo.org.co/pdf/iee/v27n2/v27n2a07.pdf

\section{Licencia Creative Commons CC BY 4.0}

Para citar este artículo: Arboleda Posada GI, Cardona Jiménez JL. Percepción de la satisfacción con la labor desempeñada y factores de motivación del personal de las instituciones prestadoras de servicios de salud(IPS) del valle de Aburrá, 2011. Rev. Gerenc. Polit. Salud. 2018;17(35). https://doi.org/10.11144/Javeriana.rgps17-35.psld 